\title{
Acoustic Realization of a Four-Dimensional Higher-Order Chern Insulator and Boundary-Modes Engineering
}

\author{
Ze-Guo Chen, Weiwei Zhu, ${ }^{*}$ Yang Tan, ${ }^{\dagger}$ Licheng Wang, ${ }^{\ddagger}$ and Guancong Ma๑ \\ Department of Physics, Hong Kong Baptist University, Kowloon Tong, Hong Kong, China
}

(Received 16 January 2020; revised 10 June 2020; accepted 15 December 2020; published 26 January 2021)

\begin{abstract}
We present a theoretical study and experimental realization of a system that is simultaneously a fourdimensional (4D) Chern insulator and a higher-order topological insulator. The system sustains the coexistence of (4-1)-dimensional chiral topological hypersurface modes (THMs) and (4-2)-dimensional chiral topological surface modes (TSMs). Our study reveals that the THMs are protected by second Chern numbers, and the TSMs are protected by a topological invariant composed of two first Chern numbers, each belonging to a Chern insulator existing in subdimensions. With the synthetic coordinates fixed, the THMs and TSMs, respectively, manifest as topological edge modes and topological corner modes (TCMs) in the real space, which are experimentally observed in a 2D acoustic lattice. These TCMs are not related to quantized polarizations, making them fundamentally distinctive from existing examples. We further show that our 4D topological system offers an effective way for the manipulation of the frequency, location, and number of TCMs, which is highly desirable for applications.
\end{abstract}

DOI: 10.1103/PhysRevX.11.011016

\section{INTRODUCTION}

The topological phase is an important development and unexplored freedom of traditional band theories [1,2]. The universality of topological phases is exemplified in a wide variety of systems, such as solid-state electronic systems [1,2], photonics [3,4], cold atoms [5], acoustics, and mechanics [6,7]. Recent studies reveal a new class of "higher-order topological insulators" (HOTIs), which refer to a $d$-dimensional topologically nontrivial system that can sustain $(d-n)$-dimensional boundary modes, with $n>1$ [8-23]. For example, 0D topological corner modes (TCMs) can be found in 2D systems. Although the studies of HOTIs have led to several significant developments, these secondorder TCMs generally do not coexist with first-order topologically protected gapless edge modes [11].

On the other hand, topological phases can also arise in parameter space that is spanned by both spatial

*Present address: Department of Physics, National University of Singapore, Singapore 117551, Republic of Singapore.

${ }^{\dagger}$ Present address: Institute of Acoustics, Department of Physics, Nanjing University, Nanjing, 210093, China.

*Present address: Department of Applied Physics, Guangdong University of Technology, Guangzhou 510090, China.

"phgcma@hkbu.edu.hk

Published by the American Physical Society under the terms of the Creative Commons Attribution 4.0 International license. Further distribution of this work must maintain attribution to the author(s) and the published article's title, journal citation, and DOI.
Subject Areas: Acoustics, Topological Insulators

(or, equivalently, reciprocal) and synthetic dimensions [24-30]. A notable example is the realization of the Hofstadter butterfly, which was originally proposed in a two-dimensional (2D) square lattice, in a 1D system by introducing one additional synthetic dimension [31]. Weyl points or semimetal, which are widely studied in $3 \mathrm{D}$ periodic systems, have also been demonstrated using a system with one spatial and two synthetic dimensions [32-34]. Synthetic dimensions also enable the investigation of systems that go beyond 3D, with the 4D quantum Hall effect being an important example $[25,35,36]$. The smart use of the extra dimensionality has led to an exciting array of novel phenomena such as the quantum Hall effect in quasicrystals [25] and topological charge pumping [29,35,36]. However, so far, the higher-order topological modes in 4D systems remain unexplored and have not been realized.

In this work, we study a 4D topological system consisting of two spatial and two synthetic dimensions. We find that the system is simultaneously a 4D Chern insulator [37] and a 4D HOTI. The system is gapless when truncated in the real space, in which case both (4-1)-dimensional chiral topological hypersurface modes (THMs) and (4-2)dimensional second-order chiral topological surface modes (TSMs) coexist. THMs are protected by the second Chern numbers of the 4D bulk bands, and TSMs are protected by nonzero combinations of first Chern numbers, each belonging to a Chern insulator existing on orthogonal subdimensions. When both synthetic coordinates are fixed, the $4 \mathrm{D}$ system is observable as $2 \mathrm{D}$ real-space systems, wherein the THMs become 1D topological edge modes (TEMs) and the TSMs manifest as OD topological corner modes (TCMs). 

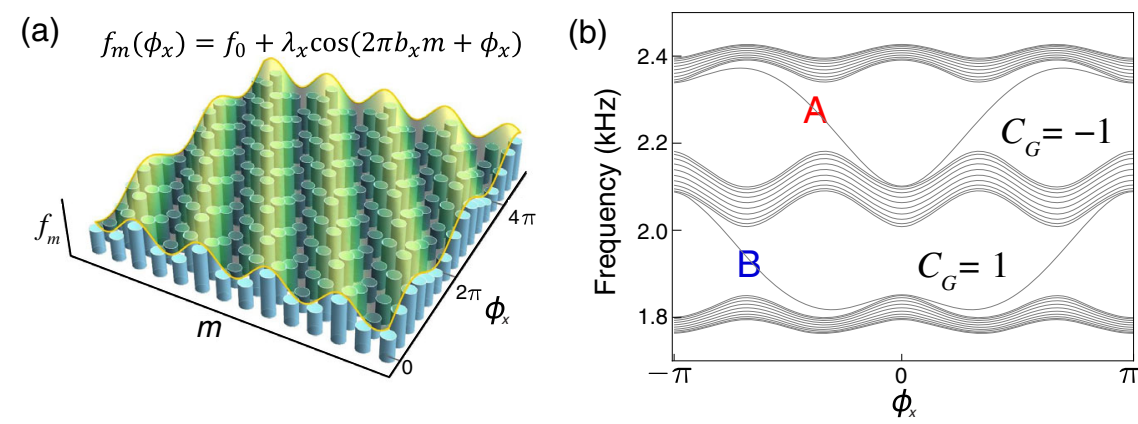

(d)

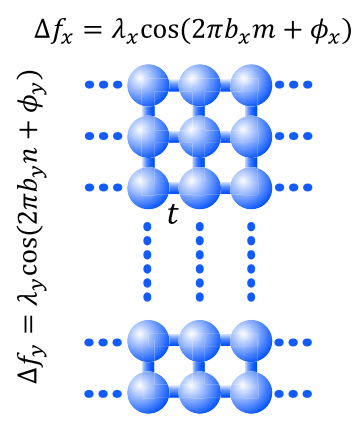

(e)

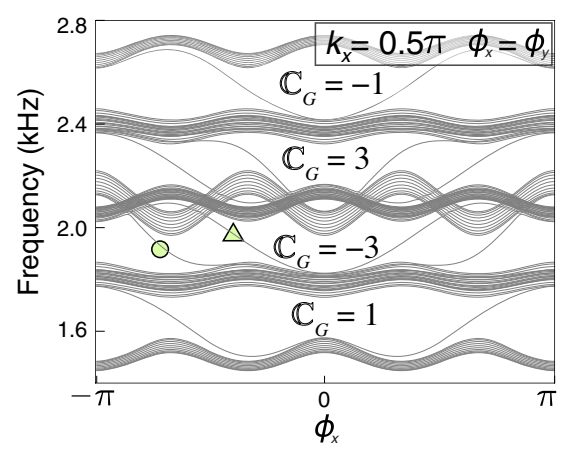

(c)

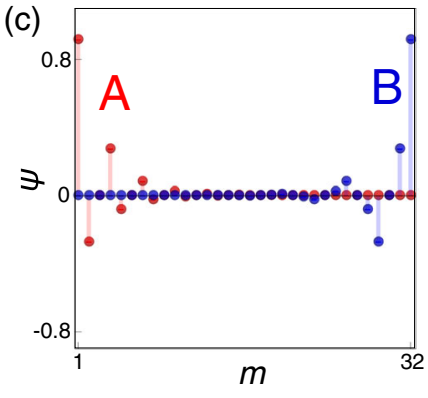

(f)

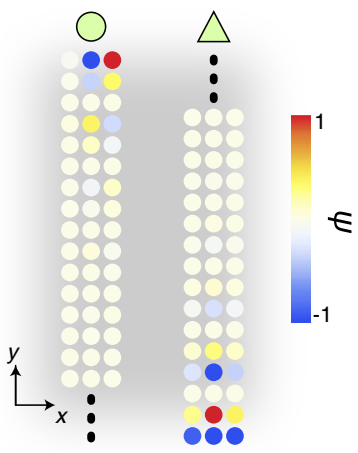

FIG. 1. 4D Chern insulator. (a) A schematic drawing of a 2D Chern insulator with one spatial and one synthetic dimension. The on-site frequency is modulated as $f_{m}\left(\phi_{x}\right)$, with $\phi_{x}$ becoming the synthetic dimension. (b) The eigenfrequencies as functions of $\phi_{x}$ calculated using the tight-binding model [Eqs. (1) and (2)]. Nonzero gap Chern numbers are marked in the band gaps. Two chiral boundary modes are shown in (c). A 4D Chern insulator can be attained using two spatial and two synthetic dimensions. Using a ribbon which is periodic in $x$ but finite in $y$ with $N_{y}=32$ sites (d), we can compute the system's eigenspectra. (e) shows the eigenspectra as a function of $\phi_{x}$ sliced at $k_{x}=0.5 \pi$ and $\phi_{x}=\phi_{y}$. The band gaps are associated with nonzero second Chern numbers as labeled. The bulk bands are closed by THMs localized at the $x$-direction edges. The real-space distributions of two THMs examples are shown in (f). All results here are obtained using a tight-binding model.

Our findings are experimentally validated using a 2D acoustic lattice. Notably, due to their 4D topological origin, the TEMs and TCMs in real-space systems are fundamentally different from previously reported cases [13-22]. On the other hand, we identify that the THMs and TSMs can be mathematically traced to the topological boundary modes of the 2D Chern insulators $[25,35,36]$. This new perspective leads to a striking capability for realizing TCMs and for manipulating their frequencies, locations, and number. Such a capability is experimentally demonstrated by the realization of two distinctive types of TCMs; one is a "separable bound state in a continuum (BIC)" [38], and the other is the realization of multiple TCMs in one corner.

\section{A 4D CHERN INSULATOR REALIZED WITH TWO SYNTHETIC DIMENSIONS}

First, we develop the theoretical model of a 4D Chern insulator and analyze its topological characteristics. Our 4D system consists of two spatial (or reciprocal) and two synthetic dimensions. To best introduce the idea, we begin by demonstrating a 2D Chern insulator with one spatial and one synthetic dimension. Consider a 1D chain of identical atoms in the $x$ direction, each coupled to its nearest neighbor through hopping $t$. The atomic chain is described by a tight-binding model

$H=\sum_{m}\left(f_{m}|m\rangle\langle m|+t| m\rangle\langle m+1|+t| m+1\rangle\langle m|\right)$,

where $|m\rangle$ is the Dirac ket for site $m$ and $t$ is the hopping constant. We enforce a modulation to the on-site eigenfrequency

$$
f_{m}\left(\phi_{x}\right)=f_{0}+\lambda_{x} \cos \left(2 \pi m b_{x}+\phi_{x}\right)
$$

where $\lambda_{x}$ is the amplitude of on-site potential and $b_{x}$ is the modulation frequency. The modulation has a phase factor $\phi_{x}$, which can be regarded as a pseudomomentum that constitutes a synthetic dimension in our system, as shown in Fig. 1(a). Here, we set $b_{x}=p / q=1 / 3$, making the system a commensurate one. We investigate a finite chain with 32 sites. The parameters used in the tightbinding models are $f_{0}=2095 \mathrm{~Hz}, t=-124.75 \mathrm{~Hz}$, and $\lambda_{x} / t=-1.9$, which are related to the acoustic system to be discussed. The procedures for determining these parameters are presented in Ref. [39]. The Hamiltonian satisfies $H\left(\phi_{x}\right)=P^{-1} H\left(-\phi_{x}\right) P$, where the nonzero element of the 
unitary operator $P$ is defined as $P_{i j}=1$ for $i+j=N+1$. This result indicates the band structure is symmetric about $\phi_{x}=0$, as shown in Fig. 1(b). Unlike the Su-SchriefferHeeger model, due to the absence of chiral symmetry for most values of $\phi_{x}$, our system generally does not have a quantized Zak phase or bulk polarizations. However, we compute the Chern numbers $C_{G}$ in the $k_{x} \phi_{x}$ plane for the first and second bulk band gaps, and both gaps have nonzero Chern numbers, which confirms that the system is a 2D Chern insulator. (See Supplemental Material [39] for more discussion.) As a result, the system is gapless, and two chiral gapless boundary modes are clearly identified [Figs. 1(b) and 1(c)]. It is noteworthy that this Chern insulator involves modulation only to on-site energy, whereas hopping $t$ remains constant. This important characteristic sets our system apart from the widely used the SuSchrieffer-Heeger model, which relies on staggered hopping but has identical on-site energy.

By incorporating two synthetic dimensions, a 4D Chern insulator can be constructed using a square lattice of nearest-coupled sites, with each on-site frequency $f_{0}$ modulated to

$$
f_{m, n}\left(\phi_{x}, \phi_{y}\right)=f_{0}+\lambda_{x} \cos \left(2 \pi b_{x} m+\phi_{x}\right)+\lambda_{y} \cos \left(2 \pi b_{y} n+\phi_{y}\right),
$$

where $m$ and $n$ label the sites, $b_{x}$ and $b_{y}$ are the modulation frequencies in the $x$ and $y$ direction, respectively, and $\phi_{x}$ and $\phi_{y}$ are the respective modulation phase factors. The system can be described by a tight-binding Hamiltonian

$$
\mathbb{H}\left(\phi_{x}, \phi_{y}\right)=\sum_{m, n}\left[\begin{array}{c}
f_{m, n}\left(\phi_{x}, \phi_{y}\right)|m, n\rangle\langle m, n| \\
+(t|m, n\rangle\langle m+1, n|+t| m, n\rangle\langle m, n+1|)+\text { H.c. }
\end{array}\right],
$$

where $|m, n\rangle$ is the Dirac ket for site $(m, n)$. Note that Eq. (3) implies that the modulation in the $x$ and $y$ directions are independent; consequently, $\phi_{x}$ and $\phi_{y}$ constitute two orthogonal dimensions. Hence, Eq. (4) describes a system living in a 4D space spanned by $\left(k_{x}, k_{y}, \phi_{x}, \phi_{y}\right)$. It also suggests that $b_{x}, \lambda_{x}, b_{y}$, and $\lambda_{y}$ can be independently tuned, which we later explore. Here, we set the modulation frequencies to be $b_{x}=b_{y}=1 / 3$ and the modulation amplitudes $\lambda_{x}=\lambda_{y}=-1.9 t$. A unit cell contains $\left(b_{x} b_{y}\right)^{-1}=9$ sites; therefore, the system has nine bulk bands. We find that these bands form five bulk band regions separated by four band gaps. The nontrivial topology of the $4 \mathrm{D}$ system is characterized by the second Chern number for bulk bands $[37,40]$

$$
\mathbb{C}_{B}=\frac{1}{32 \pi^{2}} \int d^{4} \phi \varepsilon_{i j k l} \operatorname{Tr}\left[F_{i j}^{\alpha \beta} F_{k l}^{\alpha \beta}\right]
$$

with $F_{i j}^{\alpha \beta}=\partial_{i} A_{j}^{\alpha \beta}-\partial_{j} A_{i}^{\alpha \beta}+i\left[A_{i}, A_{j}\right]^{\alpha \beta}$ and $A_{i}^{\alpha \beta}(\boldsymbol{\phi})=$ $-i\left\langle\alpha, \boldsymbol{\phi}\left|\partial / \partial \phi_{i}\right| \beta, \boldsymbol{\phi}\right\rangle$. In Eq. (5), $\varepsilon_{i j k l}$ is an antisymmetric tensor of rank 4 , and $(i, j, k, l)$ index the four dimensions: $k_{x}, k_{y}$ and $\phi_{x}, \phi_{y} . F_{i j}^{\alpha \beta}$ is the 2D Berry curvature for a state defined in pseudomomentum space $i, j$, with $\alpha, \beta$ referring to the occupied multiple bands. The second Chern numbers for $4 \mathrm{D}$ band gaps, denoted $\mathbb{C}_{G}$, can be obtained by adding the $\mathbb{C}_{B}$ of all the bands below that gap. We find that $\mathbb{C}_{G}$ for the four band gaps are $1,-3,3$, and -1 , respectively [41]. Our system is, therefore, a 4D Chern insulator.

From the bulk-surface correspondence, a nonzero second Chern number implies the existence of first-order (4-1)dimensional chiral topological modes. To investigate, we employ a 4D "ribbon" that is periodic in $x, \phi_{x}$, and $\phi_{y}$ but finite in $y$. We cut the eigenspectra at $k_{x}=0.5 \pi$, and along the line of $\phi_{x}=\phi_{y}$, the results are plotted as functions of $\phi_{x}$ shown in Fig. 1(e). It is seen that the system is indeed gapless, with its five well-defined bulk band regions connected by chiral boundary modes. In Fig. 1(f), we can see that the topological modes exponentially decay in the $y$ direction in the real space. In other words, they exist on the $k_{x} \phi_{x} \phi_{y}$ hyperplane. We therefore called them (4-1)dimensional chiral THMs. Similarly, when the 4D system is truncated in the $x$ direction, THMs are found on the $k_{y} \phi_{x} \phi_{y}$ hyperplane.

\section{A 4D HIGHER-ORDER CHERN INSULATOR}

Our system is also a 4D HOTI. To see this, we consider the same 4D system that is finite in both $x$ and $y$. The same as before, there are five bulk band regions separated by four band gaps [Fig. 2(a)]. Connecting these bulk bands are two sets of (4-1)-dimensional chiral THMs, sustained on the $k_{y} \phi_{x} \phi_{y}$ and $k_{x} \phi_{x} \phi_{y}$ hyperplanes, respectively. These are plotted in Figs. 2(b) and 2(c). Meanwhile, four (4-2)dimensional second-order TSMs are identified [Fig. 2(d)]. Note that the green surface actually contains two degenerate TSMs. The TSMs live entirely on the $\phi_{x} \phi_{y}$ plane and exponentially decay in both the $x$ and $y$ directions, as shown in Fig. 2(e). A striking observation is that these TSMs are dispersive in the two synthetic dimensions and exist entirely within the THM band gaps, making the THMs gapless. Hence, they are second-order chiral topological modes. Since the THMs are found in 4D bulk gaps, the TSMs can essentially overlap with the bulk bands in 

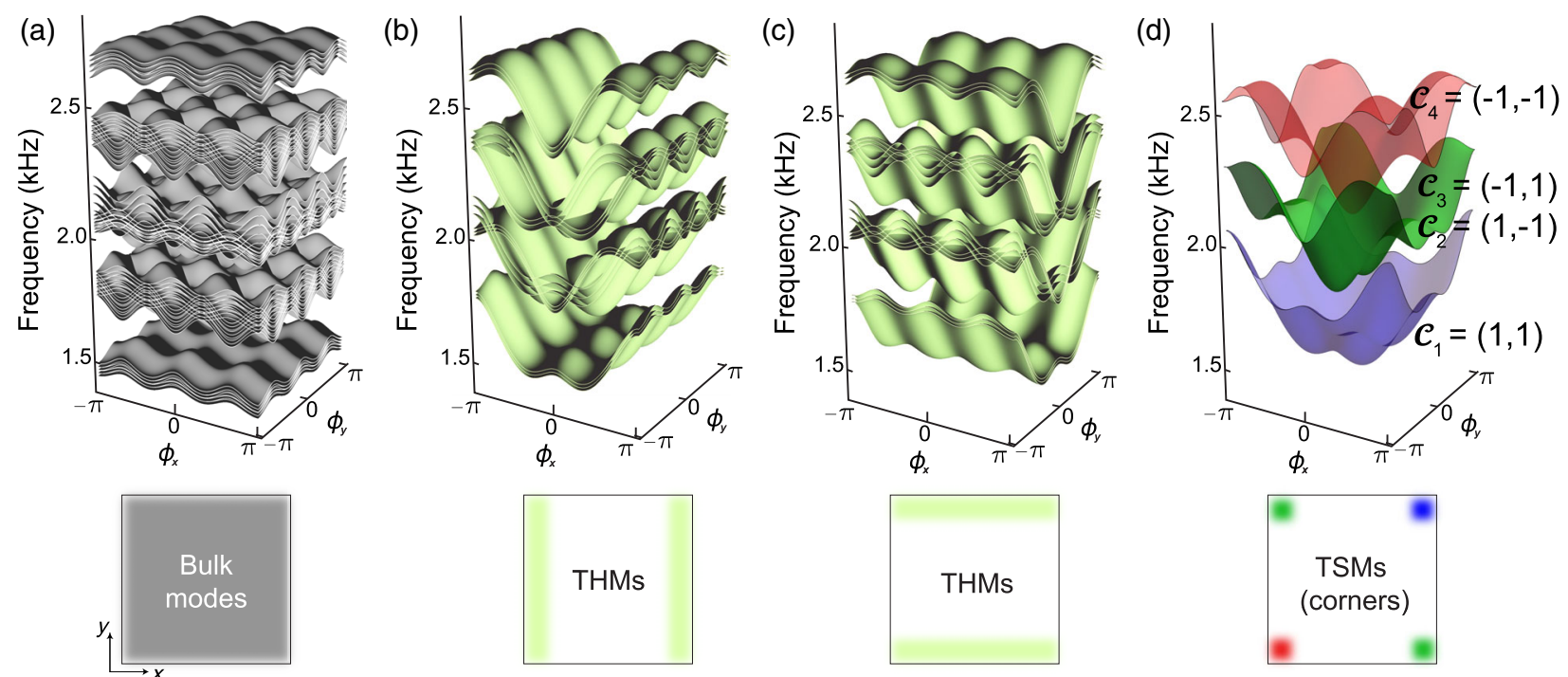

(e)
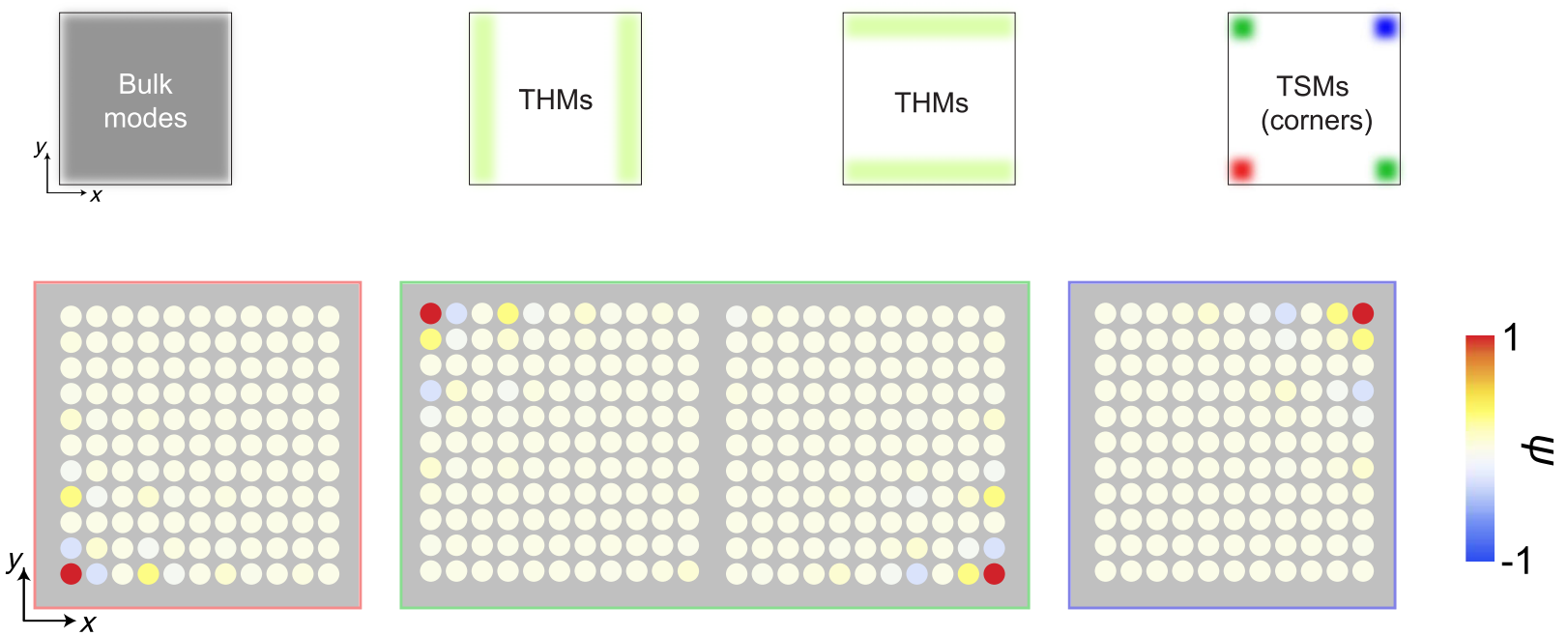

FIG. 2. Second-order gapless TSMs in a 4D Chern insulator. (a) 4D bulk modes occupy all four dimensions. Their eigenspectra are shown as functions of $\left(\phi_{x}, \phi_{y}\right)$, and they appear as 2D bulk modes in the real space (lower). (b),(c) The THMs appear in the gaps of bulk bands and live on the $k_{y} \phi_{x} \phi_{y}$ and $k_{x} \phi_{x} \phi_{y}$ hyperplanes, respectively. They are localized on the edges in the real space. (d) The TSMs are found closing the THM gaps. The TSMs are 2D modes existing on the $\phi_{x} \phi_{y}$ plane; therefore, they are observed as 0D TCMs localized at the corners in the real space. The TSMs are colored to indicate their respective locations. Note that the green sheets are two doubly degenerate states. (e) Real-space eigenfunctions of the four TSMs. The lattice here contains $11 \times 11$ sites, which is the same as the acoustic system. All results here are obtained using a tight-binding model.

frequency, implying the existence of $2 \mathrm{D}$ bound states in a 4D continuum, which we demonstrate in an experiment.

The topological nature of the TSMs can be revealed by considering the Hamiltonian of the finite system [Eq. (4)]. We observe that a finite system with $N \times N$ sites can be decomposed into two orthogonal copies of 2D Chern insulators [Eq. (1)]. Mathematically, this decomposition is expressed as

$\mathbb{H}\left(\phi_{x}, \phi_{y}\right)+f_{0} I_{N^{2}}=I_{N} \otimes H_{x}\left(\phi_{x}\right)+H_{y}\left(\phi_{y}\right) \otimes I_{N}$,

where $H_{x}\left(\phi_{x}\right)\left[H_{y}\left(\phi_{y}\right)\right]$ is the Hamiltonian of a 2D Chern system with $x(y)$ being the real dimension, $I_{N}\left(I_{N^{2}}\right)$ is an $N$ $\left(N^{2}\right)$-dimensional identity matrix, and $\otimes$ denotes the Kronecker product. The right-hand side in Eq. (6) introduces an additional on-site energy $f_{0}$ which is accounted for on the left-hand side. Equation (6) reveals the mathematical separability of $\mathbb{U}\left(\phi_{x}, \phi_{y}\right)$, which implies that
$H_{x}\left(\phi_{x}\right)$ and $H_{y}\left(\phi_{y}\right)$ exist on two orthogonal planes $k_{x} \phi_{x}$ and $k_{y} \phi_{y}$, yet these two planes do not meet. Such geometric orthogonality fundamentally roots in a 4D space. Physically, it indicates that the 4D Chern insulator can be decomposed to two independent copies of 2D Chern insulators. Equation (6) also suggests that the eigenfunctions of $\mathbb{U}\left(\phi_{x}, \phi_{y}\right)$, denoted $|\Psi\rangle$, are given by the Kronecker product of the eigenfunctions from two 2D Chern system

$$
\left|\Psi_{4 \mathrm{D}}\right\rangle=\left|\psi_{y}\right\rangle \otimes\left|\psi_{x}\right\rangle
$$

wherein $\left|\psi_{x}\right\rangle$ and $\left|\psi_{y}\right\rangle$ are the eigenfunctions of $H_{x}\left(\phi_{x}\right)$ and $H_{y}\left(\phi_{y}\right)$, respectively. The eigenvalues of $\mathbb{H}\left(\phi_{x}, \phi_{y}\right)+$ $f_{0} I_{N^{2}}$ are given by the Minkowski sum of the eigenvalues of $H_{x}\left(\phi_{x}\right)$ and $H_{y}\left(\phi_{x}\right)$. These relations make our 4D system analytical, since the separated 2D Chern system can be analytically solved [42]. Detailed discussions about the formation rules of the 4D eigenmodes and 
eigenfrequencies are presented in Ref. [39]. It follows that the TSMs are composed by the chiral boundary modes of the 2D Chern insulators existing in the subdimensions, which are protected by nonzero gap Chern numbers $C_{G}^{x}$ and $C_{G}^{y}$, respectively. As a result, the TSMs are topologically protected by a nonzero topological invariant $\mathcal{C} \equiv\left(C_{G}^{x}, C_{G}^{y}\right)$.

Although the topological invariant $\mathcal{C}$ is seemingly composed of two Chern numbers computed for 2D subsystems, it is fundamentally determined by the system's 4D topology. To see this fact, note that Eq. (6) implies that $F_{k_{x} \phi_{x}}^{\alpha \beta}$ and $F_{k_{y} \phi_{y}}^{\alpha \beta}$ are the only nonzero terms in Eq. (5). As a result, Eq. (5) is simplified to $\mathbb{C}_{B}=(1 / 2 \pi) \int d^{2} \phi F_{k_{x} \phi_{x}} \times$ $(1 / 2 \pi) \int d^{2} \phi F_{k_{y} \phi_{y}}$, i.e., the product of two nonzero first Chern numbers $[39,43]$. Since the 4D band gaps are well defined in our system, it is straightforward to consider the topology of band gaps. The second Chern number of a 4D band gap located near energy $\epsilon$ is related to the first Chern numbers of bands of 2D subsystems with energy $\epsilon_{x}+\epsilon_{y}<\epsilon[25]$ :

$$
\mathbb{C}_{G, \epsilon}=\sum_{\epsilon_{x}+\epsilon_{y}<\epsilon} C_{B, \epsilon_{x}}^{x} C_{B, \epsilon_{y}}^{y}
$$

Equation (8) helps us to build a connection between the second Chern number and the topological invariant $\sum C_{G}^{x} C_{G}^{y}$ describing the number of TSMs, where the summation is defined only in the same gap of THMs. Specifically, in our system, Eq. (8) shows that the topological invariant protecting the first TSM, i.e., $\mathcal{C}_{1}=\left(C_{G, 1}^{x}, C_{G, 1}^{y}\right)=(1,1)$, is related to the second Chern number for the first $4 \mathrm{D}$ bulk gap, $C_{G, 1}^{x} C_{G, 1}^{y}=C_{B, 1}^{x} C_{B, 1}^{y}=\mathbb{C}_{G, 1}=1$, in which the subscript numbers are the indices for band gaps. For the second and third TSMs, which are degenerate, we have, $\mathcal{C}_{2}=$ $\left(C_{G, 1}^{x}, C_{G, 2}^{y}\right)=(1,-1), \quad \mathcal{C}_{3}=\left(C_{G, 2}^{x}, C_{G, 1}^{y}\right)=(-1,1)$, and there are $C_{G, 1}^{x} C_{G, 2}^{y}+C_{G, 2}^{x} C_{G, 1}^{y}=C_{B, 1}^{x} C_{B, 1}^{y}+\left(C_{B, 1}^{x} C_{B, 1}^{y}+\right.$ $\left.C_{B, 1}^{x} C_{B, 2}^{y}+C_{B, 2}^{x} C_{B, 1}^{y}\right)=\mathbb{C}_{G, 1}+\mathbb{C}_{G, 2}=-2$. Likewise, for the fourth TSM, $\mathcal{C}_{4}=\left(C_{G, 2}^{x}, C_{G, 2}^{y}\right)=(-1,-1)$, so that $C_{G, 2}^{x} C_{G, 2}^{y}=C_{B, 1}^{x} C_{B, 1}^{y}+C_{B, 1}^{x} C_{B, 2}^{y}+C_{B, 2}^{x} C_{B, 1}^{y}+C_{B, 2}^{x} C_{B, 2}^{y}=$ $\mathbb{C}_{G, 1}+\mathbb{C}_{G, 2}-C_{B, 1}^{x} C_{B, 1}^{y}+\left(-C_{B, 1}^{x}-C_{B, 3}^{x}\right)\left(-C_{B, 1}^{y}-C_{B, 3}^{y}\right)=$ $\mathbb{C}_{G, 1}+\mathbb{C}_{G, 2}+\mathbb{C}_{G, 3}=1$.

It should be clear now that the TSMs in our system are conceptually distinctive from higher-order topological modes protected by nonzero quantized polarizations. The TSMs' topological protection is fundamentally tied to the 4D topological invariant. Such a relation between topological invariants in different dimensions is generally not present for quantized polarization. Discussion of the topological invariants for general cases with well-defined band gaps is presented in Ref. [39].

We also investigate the robustness of the THMs and TSMs against disorder. Notably, $\mathbb{C}_{G}$ remains unchanged as long as uncorrelated perturbations do not close the bulk gap, and THMs and TSMs both persist against these perturbations $[25,39]$.

\section{FORMATION RULES OF 4D EIGENMODES}

The 4D topology in our system offers rich degrees of freedom to tailor topological boundary modes in the 2D plane. The $4 \mathrm{D}$ Hamiltonian $\mathbb{U}\left(\phi_{x}, \phi_{y}\right)$ can be viewed as two compounded copies of 2D Chern insulators using the mathematical operation of the Kronecker product. Equation (7) suggests that the eigenmodes of $\mathbb{U}\left(\phi_{x}, \phi_{y}\right)$ can be constructed from the eigenmodes of $H_{x}\left(\phi_{x}\right)$ and $H_{y}\left(\phi_{y}\right)$. By considering the characteristics of the 2D Chern model's eigenmodes, we arrive at three eigenmode formation rules for the 4D system: 4D bulk modes are the product of bulk modes in both $H_{x}\left(\phi_{x}\right)$ and $H_{y}\left(\phi_{y}\right)$, i.e., $\left|\Psi_{4 \mathrm{D}}^{\text {bulk }}\right\rangle=\left|\psi_{y}^{\text {bulk }}\right\rangle \otimes\left|\psi_{x}^{\text {bulk }}\right\rangle$. THMs, if any, are the product of $2 \mathrm{D}$ bulk modes and boundary modes of $\left|\Psi_{4 \mathrm{D}}^{\mathrm{THM}}\right\rangle=$ $\left|\psi_{y}^{\text {boundary }}\right\rangle \otimes\left|\psi_{x}^{\text {bulk }}\right\rangle \quad$ or $\quad\left|\Psi_{4 \mathrm{D}}^{\mathrm{THM}}\right\rangle=\left|\psi_{y}^{\text {bulk }}\right\rangle \otimes\left|\psi_{x}^{\text {boundary }}\right\rangle$. TSMs, if any exist, are composed of the product of two boundary modes $\left|\Psi_{4 \mathrm{D}}^{\mathrm{TSM}}\right\rangle=\left|\psi_{y}^{\text {boundary }}\right\rangle \otimes\left|\psi_{x}^{\text {boundary }}\right\rangle$. Note that the formation rule for TSMs also indicates that their existence roots in the topological boundary modes in $H_{x}\left(\phi_{x}\right)$ and $H_{y}\left(\phi_{y}\right)$. This result conforms well to the fact that TSMs are topologically protected by two nonzero first Chern numbers defined in the 2D subdimensions. In addition, these formation rules suggest that the eigenfrequencies of the 4D modes are given by the Minkowski sum of those of the 2D modes. Specifically, the bulk modes' eigenfrequencies are $E_{4 \mathrm{D}}^{\text {bulk }}=E_{x}^{\text {bulk }}+E_{y}^{\text {bulk }}-f_{0}$, the THMs follow $E_{4 \mathrm{D}}^{\mathrm{THM}}=$ $E_{x}^{\text {boundary }}+E_{y}^{\text {bulk }}-f_{0} \quad$ or $\quad E_{4 \mathrm{D}}^{\mathrm{THM}}=E_{y}^{\text {boundary }}+E_{x}^{\text {bulk }}-f_{0}$, and the TSMs follow $E_{4 \mathrm{D}}^{\mathrm{TSM}}=E_{x}^{\text {boundary }}+E_{y}^{\text {boundary }}-f_{0}$. In Supplemental Material [39], we present an example of how to determine the existence and location of the 4D boundary modes using these formation rules.

In the next section, we present the experimental realization of the 4D system in acoustics. The results also showcase the formation rules, which become a convenient and flexible way to design the number, the spectral location, and the real-space location of THMs and TSMs.

\section{REALIZATION IN AN ACOUSTIC SYSTEM}

Despite the THMs and TSMs both being protected by $4 \mathrm{D}$ topological invariants, they are observable in the $2 \mathrm{D}$ realspace systems once the synthetic coordinate of $\phi_{x}, \phi_{y}$ are fixed. As clearly shown in Fig. 1(f), the THMs emerge as TEMs in the real-space lattice. Meanwhile, the TSMs manifest as 0D TCMs localized at lattice corners [Fig. 2(e)].

We use a coupled acoustic cavity system, which is a proven platform for realizing tight-binding models [44,45], for the realization of our $4 \mathrm{D}$ system. We build a $2 \mathrm{D}$ acoustic lattice with $11 \times 11$ coupled cavities. The system is shown schematically in Fig. 3(a). All cavities have an initial height 

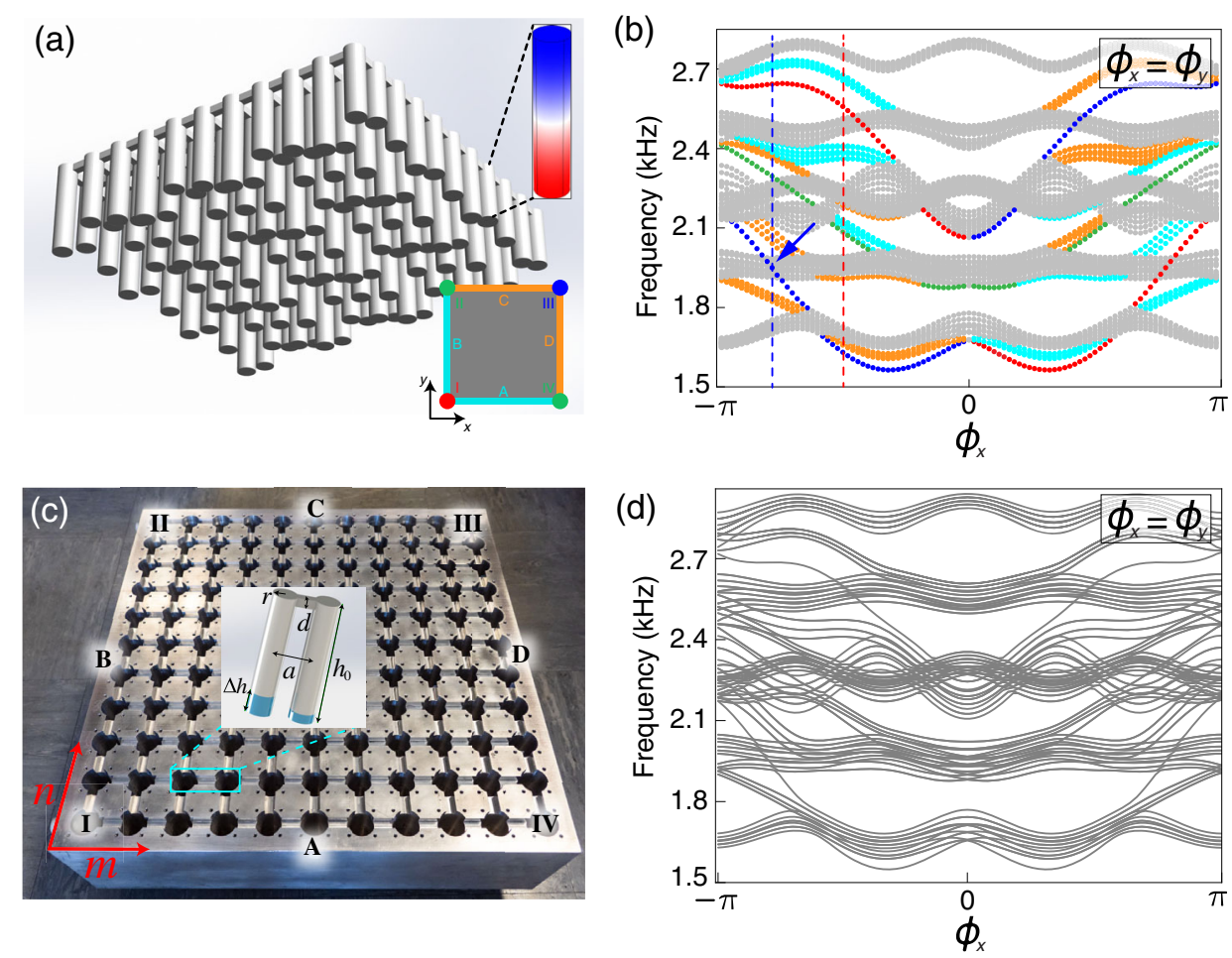

FIG. 3. Realizing the 4D system using a 2D acoustic lattice. (a) A schematic drawing of an acoustic system consisting of cavities with modulated heights. The inset shows the fundamental cavity mode which is used as the on-site orbital, where red (blue) represents positive (negative) sound pressure. In (b), the eigenspectra along $\phi_{x}=\phi_{y}$ are plotted as functions of $\phi_{x}$. The results are from finiteelement simulations. The five gray regions are bulk bands, and the cyan and orange dots are in-gap THMs. The red, green, and blue dots indicate TSMs. The colors of modes indicate their characteristics, which are represented in the inset in (a). The blue arrow in (b) indicates a TSM that overlaps with bulk bands, making it a bound state in the continuum. The red dashed line marks $\phi_{x}=-0.5 \pi$, and the blue dashed line marks $\phi_{x}=-0.78 \pi$. (c) A photograph of our acoustic lattice with $11 \times 11$ coupled cavities. To implement the two synthetic dimensions $\left(\phi_{x}, \phi_{y}\right)$, the height of each cavity is tuned by injecting a specific amount of water, as illustrated in the inset. (d) The eigenspectra along $\phi_{x}=\phi_{y}$ based on the modified tight-binding model. Excellent agreements with the results from simulations are seen.

$h_{0}=120 \mathrm{~mm}$ and a radius $r=12 \mathrm{~mm}$. The cavities are sequentially connected at the top by a square tube with a side $d=9 \mathrm{~mm}$. The outcome is a $2 \mathrm{D}$ periodic cavity lattice with a lattice constant $a=40 \mathrm{~mm}$. The first longitudinal cavity mode, which has one node in the middle of the cavity [inset in Fig. 3(a)], is chosen as the on-site orbit. This mode's natural frequency is sensitive only to the height of the cavity. Therefore, the two synthetic dimensions $\left(\phi_{x}, \phi_{y}\right)$, which modulate the on-site eigenfrequencies, can be implemented by tuning the height of each cavity. We compute the eigenspectra of the 2D acoustic lattice using the commercial finite-element solver COMSOL Multiphysics (v5.4) along the parametric line $\phi_{x}=\phi_{y}$. The result is shown in Fig. 3(b) as functions of $\phi_{x}$, in which the THMs and TSMs are colored according to their realspace locations that are shown in the inset.

We note that some topological modes extend below the first band. This extension is due to the additional on-site perturbations caused by coupling tubes, which causes the eigenspectra to deviate from the ideal tight-binding model $[39,45]$. By accounting for this perturbation, we can reproduce the acoustic band structure using a modified tight-binding model with excellent agreement, as shown in Fig. 3(d).

Experimentally, the acoustic cavity system is machined from a block of aluminum and is filled with air. An aluminum plate is fixed on the block to seal the cavities and the coupling tubes. The top of each cavity has an opening port, which is used for excitation or measurement. The ports are blocked by plugs when not in use. For the measurement of the pressure response spectra, we use a waveform generator (Keysight 33500B) to send a short pulse covering $1000-3000 \mathrm{~Hz}$ to drive a loudspeaker that is placed on top of a chosen cavity. The response signals are received by a 1/4-inch microphone (PCB Piezotronics Model-378C10) and are then recorded by a digital oscilloscope (Keysight DSO2024A). The response spectra are then obtained by performing a Fourier transform on the transient signals. The measurements are repeated for each site to obtain the sound field distribution in the entire lattice. We then extract the data points at the frequencies of interest from the spectra. The results are normalized for 
each frequency. The two synthetic dimensions are implemented by injecting a specific volume of water into each cavity to adjust its height [46]. (The water surfaces are regarded as hard walls in the simulations due to the large impedance mismatch with air.) Three groups of parameters are experimentally adopted to demonstrate topological modes of different characteristics.

\section{A. Observation of THMs and TSMs}

First, we find that at $\left(\phi_{x}, \phi_{y}\right)=(-0.5 \pi,-0.5 \pi)$, which is marked by the red dashed line in Fig. 3(b), both THMs and TSMs can be observed in the 2D lattice. We tune the acoustic lattice to this point by precisely adding a specific amount of water into each cavity. The measured results are shown in Fig. 4. In Fig. 4(a), we schematically label the edges and corners using colors and tags. First, we drive the system with a loudspeaker at the center to excite the bulk modes. The measured response spectrum is shown in Fig. 4(b) as a gray-shaded region. Five separate regions of high-pressure responses are clearly observed. We further raster-map the pressure response of all cavities at 2278 and $2538 \mathrm{~Hz}$ (marked by $f_{1}$ and $f_{2}$, respectively), as shown in Fig. 4(c). These are extended modes in both spatial dimensions, clear evidence that they are the $4 \mathrm{D}$ bulk modes. Note that, when $\phi_{x}=\phi_{y}$, the lattice possesses mirror symmetry along the line $x=y\left(M_{x=y}\right)$. This characteristic can be clearly identified in the field maps. Next, we identify that the system contains two sets of THMs, marked by cyan and orange to indicate their respective real-space locations. As THMs are localized along one spatial dimension, we can observe them by exciting the acoustic system at the corresponding edges and (a)

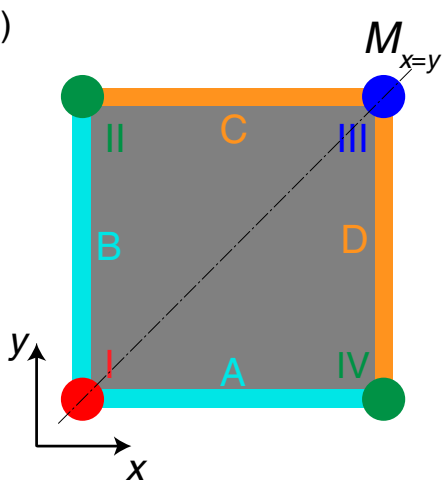

(b)

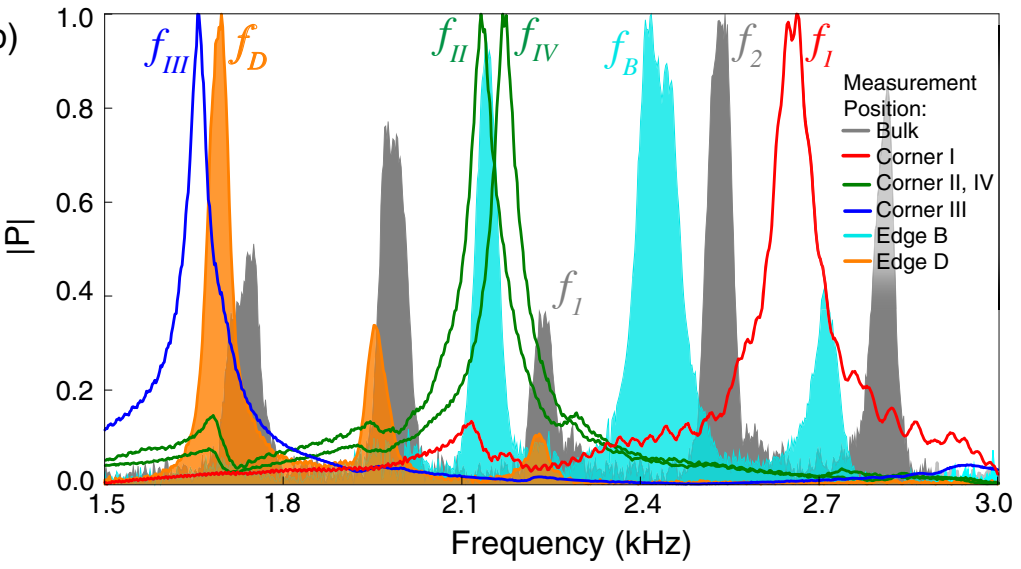

(c)

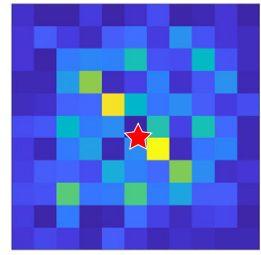

Bulk Mode, $f_{l}$

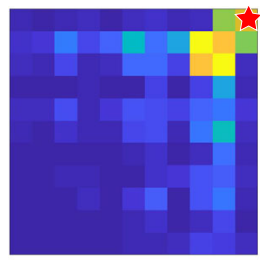

Bulk Mode, $f_{2}$ (d)

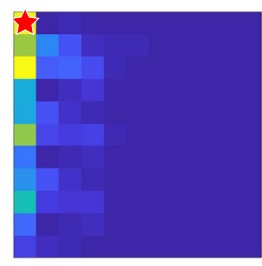

THMs, $f_{B}$

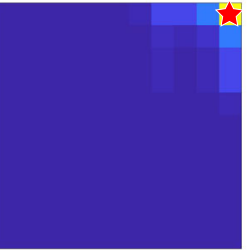

TSM, $f_{I I I}$

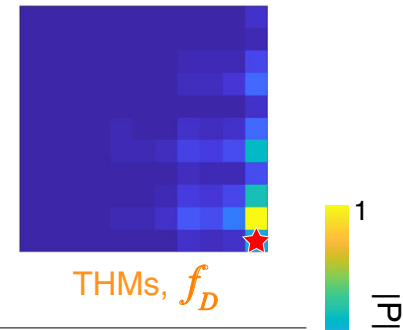

$\bar{\tau}$ (e)

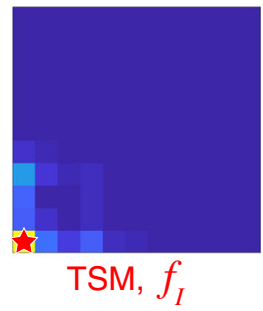

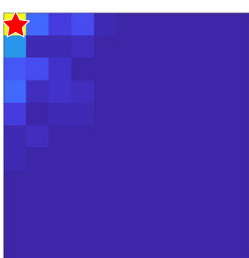

TSM, $f_{I I}$

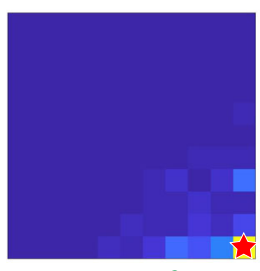

TSM, $f_{I V}$

FIG. 4. Observation of THMs and TSMs. Here, the system is at $\left(\phi_{x}, \phi_{y}\right)=(-0.5 \pi,-0.5 \pi)$. (a) A schematic drawing of the system, wherein the corners and edges are color labeled. Note that the system has mirror symmetry $M_{x=y}$. (b) The pressure response spectra. The gray areas represent the bulk response; the orange- and cyan-shaded areas are the edge responses; the four curves each represent the response at the correspondingly colored corner. The spatial field maps are shown when the system is excited in the bulk (c), at the edges (d), and at the corners (e) at the indicated frequencies. The red stars in (c)-(e) mark the excitation position. In (d), the field maps are confined at the excitation edges, indicating the observation of THMs in real space as TEMs. In (e), the modes are strongly localized at the excitation corners, indicating the existence of TSMs in real space. 
measure their response spectra, which are shown in Fig. 4(b). Three peaks are seen for both edges $A$ and $B$ (cyan) and edges $C$ and $D$ (orange), which are consistent with our prediction as well as the simulation results in Fig. 3(b). Two cases of the spatial distributions of these modes are shown in Fig. 4(d), which clearly show that these are TEMs localized at the sample's edges, which agree well with our prediction. The TSMs, marked by red, blue, and green in Fig. 4(a), are 0D modes localized at the corners of the $2 \mathrm{D}$ lattice. By placing the source at the corresponding corner, we observe only one sharp resonant peak at each corner [Fig. 4(b)]. Spatial pressure maps at each peak frequency further confirm that these modes are strongly localized at the corner and decay rapidly into the bulk [Fig. 4(e)]. We note that the states at corners II and IV are ideally degenerate, owing to the system's mirror symmetry $M_{x=y}$ (along $x=y$ ). In the measured results, the two corresponding resonant peaks slightly mismatch in frequency [Fig. 4(b)]. We attribute such discrepancy to experimental errors, which may cause $\phi_{x}$ and $\phi_{y}$ to deviate from the ideal value. This is also a strong evidence that the existence of the THMs and TSMs are robust against disorders. In summary, the results confirm that the system possesses both TEMs and TCMs, which validates that the 4D system simultaneously supports both first-order THMs and second-order TSMs.

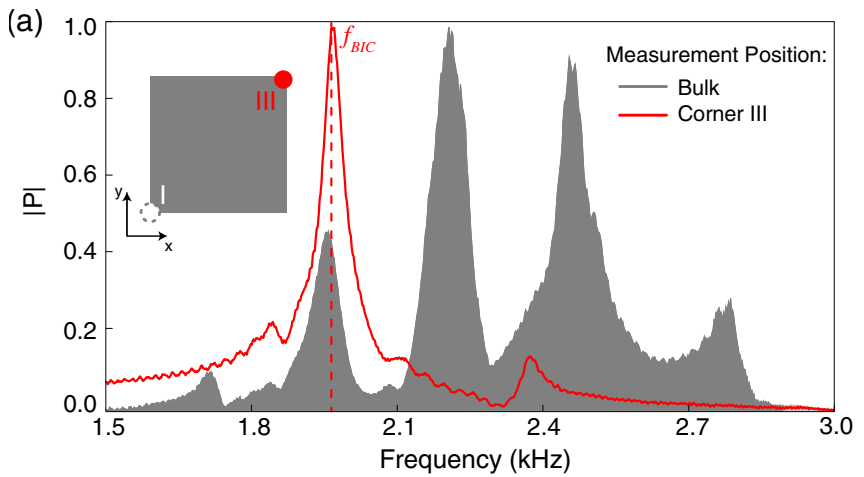

(b)

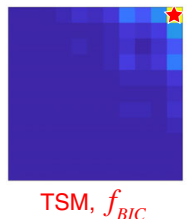

(c)

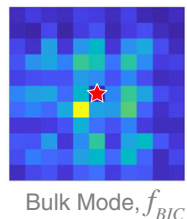

(d)

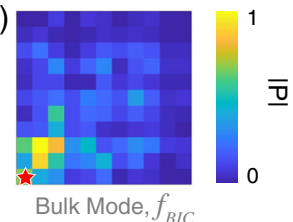

FIG. 5. Using TSM to realize BIC in real space. (a) The pressure responses measured at corner III (red) and in the bulk (gray region). A single peak at $f_{\mathrm{BIC}}=2060 \mathrm{~Hz}$ is seen for the corner response, which overlaps with the second bulk band. (b) The spatial field map at $f_{\text {BIC }}$ when the source is at corner III. A highly localized corner mode is seen. (c) Excited at $f_{\text {BIC }}$ by a source at the center, extended modes occupying the entire bulk are seen. (d) In comparison, when the system is pumped at $f_{\mathrm{BIC}}$ by a source placed at corner I, the field map indicates extended modes. The inset in (a) is a schematic drawing of the 2D acoustic lattice with the corners are color tagged.

\section{B. TSM as a bound state in the continuum}

The fact that TSMs in our system are chiral modes closing the gaps of THMs has two implications. First, the TSMs are dispersive in the synthetic coordinates; second, as THMs are entirely in the 4D bulk gaps, the TSMs can overlap with the bulk bands in frequency, becoming bound states in the bulk continuum. An example can be seen near $\left(\phi_{x}, \phi_{y}\right)=(-0.78 \pi,-0.78 \pi)$, which is marked by the blue dashed line in Fig. 3(b). Since TSMs are TCMs in real space, they are observable as corner-mode BICs.

We tune the acoustic system to this parameter point by adjusting the amount water in each cavity. Figure 5(a) shows the pressure response spectra of this case. When the

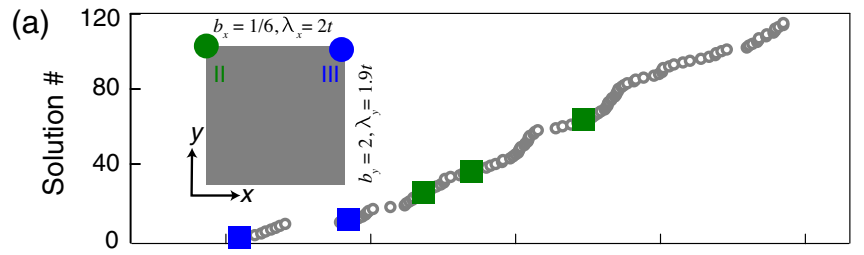

(b)

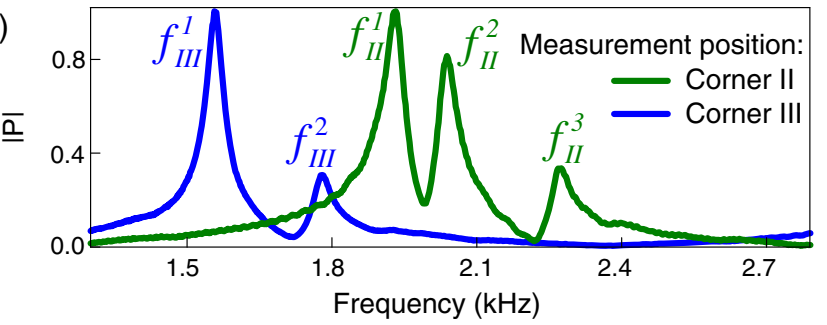

(c)

TSMs at corner II
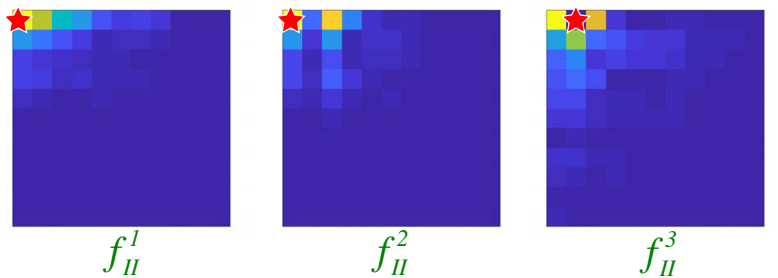

(d)

TSMs at corner III
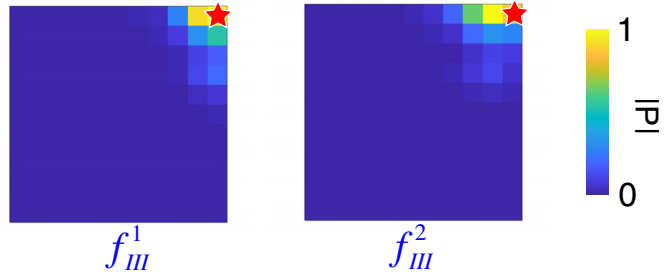

FIG. 6. Exploiting the TSMs for the realization of multiple TCMs at the same corner. (a) Eigenspectrum of a 4D acoustic system with $b_{x}=1 / 6, b_{y}=1 / 3$ at $\left(\phi_{x}, \phi_{y}\right)=(-0.6 \pi,-0.28 \pi)$. (b) The measured corner responses have three peaks for corner II (green) and two peaks for corner III (blue). The peak frequencies are consistent with the eigenfrequencies found in finite-element simulation. (c) and (d) are field maps of the three TSMs at corner II and the two TSMs at corner III, respectively. The red stars mark the source position in each case. Note that the third TSM at corner II is excited with a source located one site away from the corner. 
excitation is at corner III, one single response peak is seen at $f_{\mathrm{BIC}}=2060 \mathrm{~Hz}$ (red curve). This peak spectrally overlaps with the second bulk band (gray regions). We then place the source at corner III to excite at $f_{\text {BIC }}$ and obtain the field maps. A highly localized corner mode is clearly seen [Fig. 5(b)]. In contrast, bulk modes are excited at the same frequency when the source is in the center [Fig. 5(c)] or at corner I [Fig. 5(d)]. These results unambiguously show that the mode at corner III at $f_{\mathrm{BIC}}$ is a BIC $[38,47]$.

\section{Multiple TSMs localized at the same corner}

The characteristics of THMs and TSMs are fully revealed only when considering all four dimensions; they are, nevertheless, observable as TEMs and TCMs in real space. This fact means that the real-space descendant system can also be regarded as a new type of 2D HOTI that simultaneously supports TEMs and TCMs. Moreover, the $4 \mathrm{D}$ system brings extra degrees of freedom in the manipulation of the TEMs and TCMs.

To show the unique advantage of our system, we set $b_{x}=$ $1 / 6$ and $\lambda_{x}=-2 t$ while keeping $b_{y}=1 / 3$ and $\lambda_{y}=-1.9 t$. We analyze the point $\left(\phi_{x}, \phi_{y}\right)=(-0.6 \pi,-0.28 \pi)$ and find a total of five TSMs in this system, as shown in the eigenspectrum in Fig. 6(a). In the 2D lattice, three TSMs are localized at corner II and the other two localized at corner III. These are shown in Fig. 6(b). We validate these findings in our acoustic system. Our results show strong evidence for the existence of all five TSMs as corner modes. Three (two) resonant peaks are clearly seen when the system is excited at corner II (III) [Fig. 6(b)]. The field distributions at the peak frequencies [Figs. 6(c) and 6(d)] indicate localized modes at their respective corners.

\section{The absence of TSMs at $\left(\phi_{x}, \phi_{y}\right)=(-0.5 \pi, 0)$}

Here, we return to $b_{x}=b_{y}=1 / 3$. In Fig. 1(b), we can identify that, at $\phi_{x}=0$, the 1D chain does not possess a welldistinguished boundary mode. By choosing $\left(\phi_{x}, \phi_{y}\right)=$ $(-0.5 \pi, 0)$ for the 4D system, from our formation rules, we know that: first, THMs can be observed only at edges $B$ and $D$ in the real-space lattice; second, TSM shall be absent.

Our predictions are experimentally verified. Note that, when $\phi_{y}=0$, the system has mirror symmetry $M_{y}$. The response spectra measured in the bulk and at corners I and III [Fig. 7(a)] are shown in Fig. 7(b). The corner responses each have two resonant peaks. Note that response peaks do not necessarily indicate corner modes. In this case, they are due to THMs at either joining edge, which is confirmed by the raster-maps of the sound fields, as shown in Figs. 7(c) and 7(d). Note that the sources at corners I and III excite modes that are confined along edges $B$ and $D$, respectively, whereas no modes along edges $A$ and $C$ are seen. The symmetry $M_{y}$ suggests the same be observed for corners II and IV. These observations align well with the predictions from the formation rules.

\section{E. The absence of THMs and TSMs at $\left(\phi_{x}, \phi_{y}\right)=(0,0)$}

The above discussion and results suggest that, by choosing $\left(\phi_{x}, \phi_{y}\right)=(0,0)$, both THMs and TSMs shall be absent. This result is demonstrated in the results shown (a)

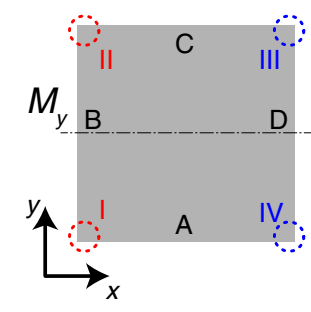

(c)

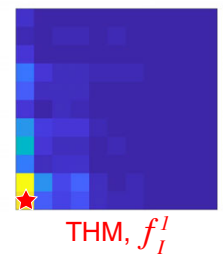

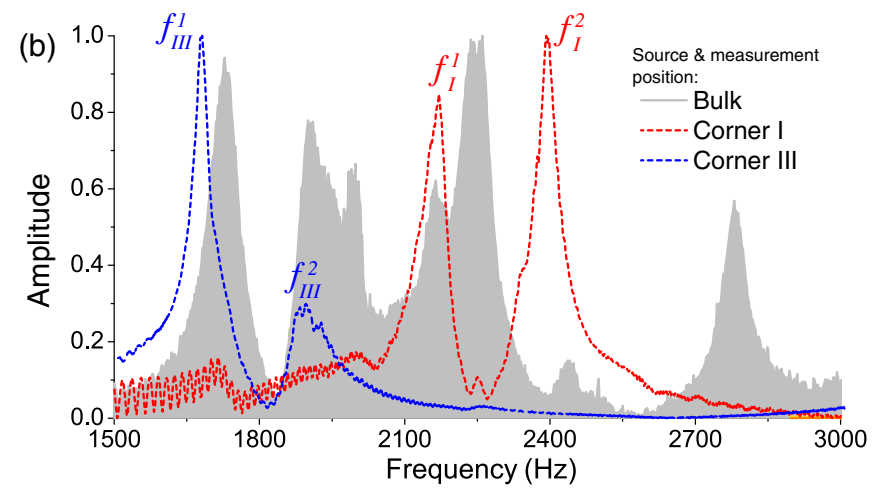

(d)

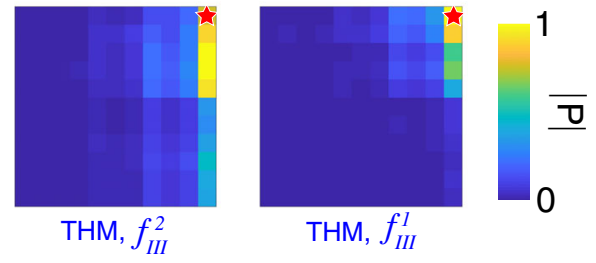

FIG. 7. The experimental results at $\left(\phi_{x}, \phi_{y}\right)=(-0.5 \pi, 0)$. (a) A schematic drawing of the system, with the bulk, edges, and corners marked in different colors. (b) The gray-colored region is pressure response in the bulk; the red and blue dashed curves are, respectively, the response measured at corner I and corner III. (c) The field maps when excited by a source located at corner I at the two frequencies as marked in (b). The distributions suggest THMs are found only at edge $B$. (d) The field maps at two frequencies from the peak of the corner III response spectrum. The distributions suggest THMs localized at edge $D$. The red stars mark the source positions. 
(a)

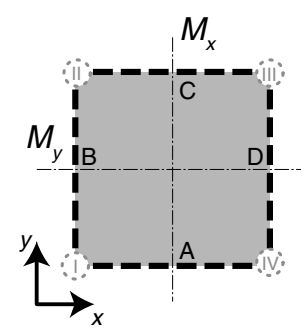

(c)

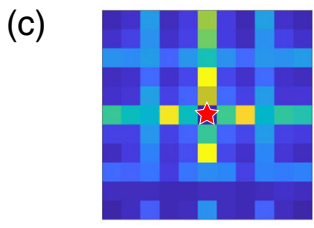

(d)

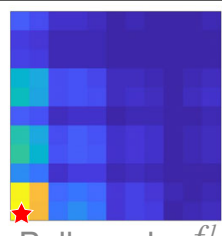

Bulk mode, $f_{I}^{I}$
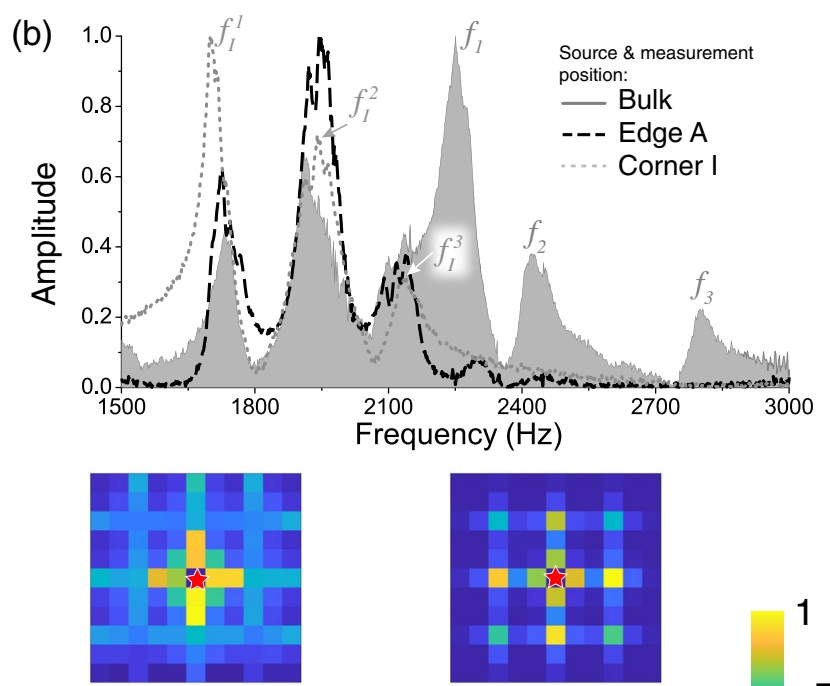

Bulk mode, $f_{2}$

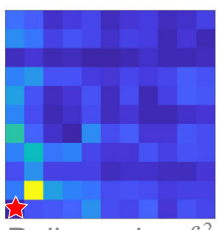

Bulk mode, $f_{I}^{2}$

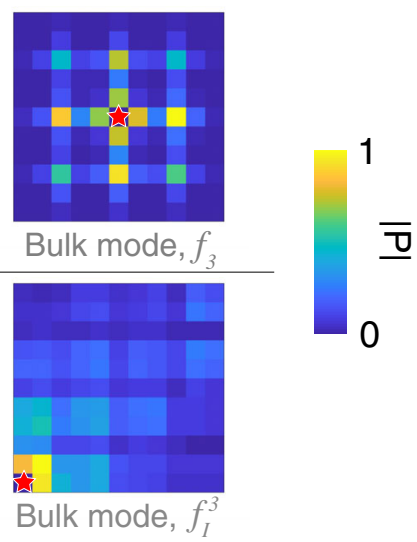

FIG. 8. Experimental results at $\left(\phi_{x}, \phi_{y}\right)=(0,0)$. (a) A schematic drawing of the system. (b) The pressure responses with different excitation and measurement positions as indicated in the legend. (c) The field maps of the bulk modes at frequencies as indicated. (d) The field maps of the bulk modes at frequencies as indicated, with the source at corner I. The red stars in (c) and (d) indicate the source positions.

in Fig. 8. Note that the system has mirror symmetry $M_{x}$ and $M_{y}$, as shown in Fig. 8(a). Such mirror symmetries are clearly visible in the raster-maps shown in Fig. 8(c), which show the bulk modes with the source placed at the center of the lattice. We take advantage of the mirror symmetries and examine only corner I and edge $A$ to check for THMs and TSMs. Three peaks measured at corner I are found at $f_{I}^{1}$, $f_{I}^{2}$, and $f_{I}^{3}$, respectively [the light-gray dotted curve in Fig. 8(b)], which largely overlaps in their frequencies with the three peaks obtained at edge $A$, which hints that they may be due to the same set of modes. The field maps further confirm that these peaks are not localized modes but rather extended bulk modes. The predictions from the eigenmode formation rules are again experimentally validated.

\section{DISCUSSION AND CONCLUSIONS}

Our 4D topological system simultaneously sustains firstorder THMs and second-order TSMs. Both the THMs and TSMs are gapless. In the real-space descendant system with the synthetic coordinates fixed, the TSMs become the TCMs to be observable in experiments. Hence, these TCMs are fundamentally different from those reported previously, which are typically the consequence of nonzero corner charges induced by quantized polarizations. In contrast, the topological invariant protecting our TCMs is unveiled only by ascending to 4D. The topological invariant, which consists of two first Chern numbers, each responsible for a Chern insulator in a $2 \mathrm{D}$ plane, can be meaningfully defined only in a $4 \mathrm{D}$ hyperspace. Its $4 \mathrm{D}$ origin is further confirmed by its close tie to the second Chern numbers of the 4D gap, shown in Sec. III. On the other hand, despite the real-space system being a square or rectangular lattice, it does not possess any crystalline symmetry for most values of $\phi_{x, y}$. A direct consequence is that bulk polarizations are not quantized and cannot serve as a topological invariant in our system. This constraint again fundamentally distinguishes our system from existing HOTIs.

The rich degrees of freedom offered by the 4D topology lead to a powerful recipe that brings unprecedented capabilities to tailor higher-order topological modes. In particular, Eq. (7) and the formation rules (Sec. IV) are new routes to convenient, versatile control of TEMs and TCMs in real space. Such a capability is desirable for applications utilizing these modes, such as wave steering and wave localizations.

In conclusion, we have demonstrated with both theory and acoustic experiments a 4D Chern and HOTI. Our work expands the concept of HOTIs to 4D systems. The ideas demonstrated in this paper are general and can be adapted for other types of waves, such as mechanical systems, electromagnetism, and photonics. We also expect rich 
phenomena to be discovered by the clever design of the modulation functions or by using other types of topologically nontrivial models. It can also be useful for building systems in even higher dimensions.

\section{ACKNOWLEDGMENTS}

The authors thank Shiqiao Wu and Weiyuan Tang for sample preparation. Z.-G. C. and G. M. thank C. T. Chan, Zhao-Qing Zhang, Cheng He, Ruoyang Zhang, and Biao Yang for fruitful discussions. This work is supported by National Science Foundation of China (NSFC) Excellent Young Scientist Scheme (Hong Kong \& Macau) (No. 11922416) and NSFC Youth Program (No. 11802256), Hong Kong Research Grants Council (GRF 12302420, GRF 12300419, ECS 22302718, and C6013-18G), and Hong Kong Baptist University (RCSGT2/18-19/SCI/006).

[1] X.-L. Qi and S.-C. Zhang, Topological Insulators and Superconductors, Rev. Mod. Phys. 83, 1057 (2011).

[2] M.Z. Hasan and C. L. Kane, Colloquium: Topological Insulators, Rev. Mod. Phys. 82, 3045 (2010).

[3] L. Lu, J. D. Joannopoulos, and M. Soljacic, Topological Photonics, Nat. Photonics 8, 821 (2014).

[4] T. Ozawa, H. M. Price, A. Amo, N. Goldman, M. Hafezi, L. Lu, M. C. Rechtsman, D. Schuster, J. Simon, and O. Zilberberg, Topological Photonics, Rev. Mod. Phys. 91, 015006 (2019).

[5] N. R. Cooper, J. Dalibard, and I. B. Spielman, Topological Bands for Ultracold Atoms, Rev. Mod. Phys. 91, 015005 (2019).

[6] G. Ma, M. Xiao, and C. T. Chan, Topological Phases in Acoustic and Mechanical Systems, Nat. Rev. Phys. 1, 281 (2019).

[7] X. Zhang, M. Xiao, Y. Cheng, M.-H. Lu, and J. Christensen, Topological Sound, Commun. Phys. 1, 97 (2018).

[8] W. A. Benalcazar, B. A. Bernevig, and T. L. Hughes, Quantized Electric Multipole Insulators, Science 357, 61 (2017).

[9] J. Langbehn, Y. Peng, L. Trifunovic, F. von Oppen, and P. W. Brouwer, Reflection-Symmetric Second-Order Topological Insulators and Superconductors, Phys. Rev. Lett. 119, 246401 (2017).

[10] Z. Song, Z. Fang, and C. Fang, d-2-Dimensional Edge States of Rotation Symmetry Protected Topological States, Phys. Rev. Lett. 119, 246402 (2017).

[11] M. Ezawa, Higher-Order Topological Insulators and Semimetals on the Breathing Kagome and Pyrochlore Lattices, Phys. Rev. Lett. 120, 026801 (2018).

[12] S. Imhof, C. Berger, F. Bayer, J. Brehm, L. W. Molenkamp, T. Kiessling, F. Schindler, C. H. Lee, M. Greiter, and T. Neupert et al., Topolectrical-Circuit Realization of Topological Corner Modes, Nat. Phys. 14, 925 (2018).

[13] C. W. Peterson, W. A. Benalcazar, T. L. Hughes, and G. Bahl, A Quantized Microwave Quadrupole Insulator with Topologically Protected Corner States, Nature (London) 555, 346 (2018).
[14] F. Schindler, A. M. Cook, M. G. Vergniory, Z. Wang, S. S. P. Parkin, B. A. Bernevig, and T. Neupert, Higher-Order Topological Insulators, Sci. Adv. 4, eaat0346 (2018).

[15] M. Serra-Garcia, V. Peri, R. Süsstrunk, O. R. Bilal, T. Larsen, L. G. Villanueva, and S. D. Huber, Observation of a Phononic Quadrupole Topological Insulator, Nature (London) 555, 342 (2018).

[16] B.-Y. Xie, H.-F. Wang, H.-X. Wang, X.-Y. Zhu, J.-H. Jiang, M. H. Lu, and Y.F. Chen, Second-Order Photonic Topological Insulator with Corner States, Phys. Rev. B 98, 205147 (2018).

[17] H. Xue, Y. Yang, F. Gao, Y. Chong, and B. Zhang, Acoustic Higher-Order Topological Insulator on a Kagome Lattice, Nat. Mater. 18, 108 (2019).

[18] Z.-G. Chen, C. Xu, R. Al Jahdali, J. Mei, and Y. Wu, Corner States in a Second-Order Acoustic Topological Insulator as Bound States in the Continuum, Phys. Rev. B 100, 075120 (2019).

[19] X. Ni, M. Weiner, A. Alù, and A. B. Khanikaev, Observation of Higher-Order Topological Acoustic States Protected by Generalized Chiral Symmetry, Nat. Mater. 18, 113 (2019).

[20] Z. Zhang, H. Long, C. Liu, C. Shao, Y. Cheng, X. Liu, and J. Christensen, Deep-Subwavelength Holey Acoustic SecondOrder Topological Insulators, Adv. Mater. 31, 1904682 (2019).

[21] W. Ma, L. Zhou, Q. Zhang, M. Li, C. Cheng, J. Geng, X. Rong, F. Shi, J. Gong, and J. Du, Experimental Observation of a Generalized Thouless Pump with a Single Spin, Phys. Rev. Lett. 120, 120501 (2018).

[22] J. Noh, W. A. Benalcazar, S. Huang, M. J. Collins, K. P. Chen, T. L. Hughes, and M. C. Rechtsman, Topological Protection of Photonic Mid-Gap Defect Modes, Nat. Photonics 12, 408 (2018).

[23] X. Zhang, H.-X. Wang, Z.-K. Lin, Y. Tian, B. Xie, M.-H. Lu, Y.-F. Chen, and J.-H. Jiang, Second-Order Topology and Multidimensional Topological Transitions in Sonic Crystals, Nat. Phys. 15, 582 (2019).

[24] L.-J. Lang, X. Cai, and S. Chen, Edge States and Topological Phases in One-Dimensional Optical Superlattices, Phys. Rev. Lett. 108, 220401 (2012).

[25] Y.E. Kraus, Z. Ringel, and O. Zilberberg, FourDimensional Quantum Hall Effect in a Two-Dimensional Quasicrystal, Phys. Rev. Lett. 111, 226401 (2013).

[26] Y.E. Kraus and O. Zilberberg, Topological Equivalence between the Fibonacci Quasicrystal and the Harper Model, Phys. Rev. Lett. 109, 116404 (2012).

[27] W. Zhu, X. Fang, D. Li, Y. Sun, Y. Li, Y. Jing, and H. Chen, Simultaneous Observation of a Topological Edge State and Exceptional Point in an Open and Non-Hermitian Acoustic System, Phys. Rev. Lett. 121, 124501 (2018).

[28] D. J. Apigo, W. Cheng, K. F. Dobiszewski, E. Prodan, and C. Prodan, Observation of Topological Edge Modes in a Quasiperiodic Acoustic Waveguide, Phys. Rev. Lett. 122, 095501 (2019).

[29] Y. Long and J. Ren, Floquet Topological Acoustic Resonators and Acoustic Thouless Pumping, J. Acoust. Soc. Am. 146, 742 (2019).

[30] L. Yuan, Q. Lin, M. Xiao, and S. Fan, Synthetic Dimension in Photonics, Optica 5, 1396 (2018). 
[31] X. Ni, K. Chen, M. Weiner, D. J. Apigo, C. Prodan, A. Alù, E. Prodan, and A. B. Khanikaev, Observation of Hofstadter Butterfly and Topological Edge States in Reconfigurable Quasi-Periodic Acoustic Crystals, Commun. Phys. 2, 55 (2019).

[32] Q. Wang, M. Xiao, H. Liu, S. Zhu, and C. T. Chan, Optical Interface States Protected by Synthetic Weyl Points, Phys. Rev. X 7, 031032 (2017).

[33] X. Fan, C. Qiu, Y. Shen, H. He, M. Xiao, M. Ke, and Z. Liu, Probing Weyl Physics with One-Dimensional Sonic Crystals, Phys. Rev. Lett. 122, 136802 (2019).

[34] F. Zangeneh-Nejad and R. Fleury, Experimental Observation of the Acoustic Z2 Weyl Semimetallic Phase in Synthetic Dimensions, Phys. Rev. B 102, 064309 (2020).

[35] M. Lohse, C. Schweizer, H. M. Price, O. Zilberberg, and I. Bloch, Exploring 4D Quantum Hall Physics with a $2 D$ Topological Charge Pump, Nature (London) 553, 55 (2018).

[36] O. Zilberberg, S. Huang, J. Guglielmon, M. Wang, K. P. Chen, Y. E. Kraus, and M. C. Rechtsman, Photonic Topological Boundary Pumping as a Probe of 4D Quantum Hall Physics, Nature (London) 553, 59 (2018).

[37] S.-C. Zhang and J. Hu, A Four-Dimensional Generalization of the Quantum Hall Effect, Science 294, 823 (2001).

[38] C. W. Hsu, B. Zhen, A. D. Stone, J. D. Joannopoulos, and M. Soljačić, Bound States in the Continuum, Nat. Rev. Mater. 1, 16048 (2016).

[39] See Supplemental Material at http://link.aps.org/ supplemental/10.1103/PhysRevX.11.011016 for additional details on the relation between the tight-binding models and our acoustic structures; parameter discussion; the topological characterizations and robustness discussion of the THMs and TSMs; the eigenmode formation rules; and the simulation results of the boundary modes.

[40] X.-L. Qi, T. L. Hughes, and S.-C. Zhang, Topological Field Theory of Time-Reversal Invariant Insulators, Phys. Rev. B 78, 195424 (2008).

[41] M. Mochol-Grzelak, A. Dauphin, A. Celi, and M. Lewenstein, Efficient Algorithm to Compute the Second Chern Number in Four Dimensional Systems, Quantum Sci. Technol. 4, 014009 (2018).

[42] A. M. Marques and R. G. Dias, Analytical Solution of Open Crystalline Linear 1D Tight-Binding Models, J. Phys. A 53, 075303 (2020).

[43] X. Zhang, Y. Chen, Y. Wang, Y. Liu, J. Y. Lin, N. C. Hu, B. Guan, and C. H. Lee, Entangled Four-Dimensional Multicomponent Topological States from Photonic Crystal Defects, Phys. Rev. B 100, 041110(R) (2019).

[44] Y.-X. Xiao, G. Ma, Z.-Q. Zhang, and C. T. Chan, Topological Subspace-Induced Bound State in the Continuum, Phys. Rev. Lett. 118, 166803 (2017).

[45] Z.-G. Chen, L. Wang, G. Zhang, and G. Ma, Chiral Symmetry Breaking of Tight-Binding Models in Coupled Acoustic-Cavity Systems, Phys. Rev. Applied 14, 024023 (2020).

[46] N. Kaina, F. Lemoult, M. Fink, and G. Lerosey, Negative Refractive Index and Acoustic Superlens from Multiple Scattering in Single Negative Metamaterials, Nature (London) 525, 77 (2015).

[47] M. Robnik, A Simple Separable Hamiltonian Having Bound States in the Continuum, J. Phys. A 19, 3845 (1986). 